\title{
A TOPOLOGICAL DISK IN A 4-MANIFOLD CAN BE APPROXIMATED BY PIECEWISE LINEAR DISKS
}

\author{
BY GERARD A. VENEMA
}

Communicated by T. A. Chapman, December 2, 1976

Several approximation theorems for embeddings of codimension 2 cells are announced here and the proofs are outlined. More detailed proofs will appear elsewhere [5].

1. Introduction. Our main theorem asserts that any topological embedding of a disk (2-cell) in a piecewise linear 4-manifold can be approximated arbitrarily closely by locally flat, piecewise linear embeddings. For codimension 2 cells in general, we do not prove as strong a theorem. If $M^{n}$ is a piecewise linear (PL) manifold and the topological embedding $D: I^{n-2} \rightarrow M^{n}$ has the property that there is some open set $U \subset I^{n-2}$ such that $D \mid U$ can be $\epsilon$-approximated for every $\epsilon>0$, then we show that $D$ can be $\epsilon$-approximated for every $\epsilon>0$. A corollary is that a piecewise linear, codimension 2 cell can be approximated by locally flat $(n-2)$-cells in all dimensions.

If $D: I^{n-2} \rightarrow M^{n}$ is the topological embedding, the approximation can be chosen to agree with $D$ on $\partial I^{n-2}$ in both the theorems providing that $D / \partial I^{n-2}$ is PL and $D\left(\partial I^{n-2}\right) \subset$ Int $M$. This can be accomplished simply by pushing the boundary of the approximation to the boundary of $D$ with a small ambient isotopyusing [2] in case $n=4$ and [3] in case $n \geqslant 5$. However, if $D\left(\partial I^{n-2}\right) \subset \partial M$, the approximation cannot agree with $D$ on the boundary. It is also not possible to replace $\epsilon>0$ with a function $\epsilon(x)>0$ with $\epsilon(x) \rightarrow 0$ as $x \rightarrow \partial M$. For example, the cone over the trefoil knot in the boundary of $E_{+}^{4}$ cannot be approximated in this way. In fact, the trefoil knot does not bound any locally flat PL disk in $E_{+}^{4}[4]$.

2. Statement of the theorems.

THEOREM 1. If $D: I^{2} \rightarrow M^{4}$ is a topological embedding of a disk into a $P L$ 4-manifold, then $D$ can be e-approximated by a locally flat PL embedding $E: I^{2} \rightarrow M^{4}$ for every $\epsilon>0$.

THEOREM 2. Suppose $M^{n}$ is a PL n-manifold and $D: I^{n-2} \rightarrow M^{n}$ is a topological embedding. If there exists an open set $U \subset I^{n-2}$ such that $D \mid U$ has

AMS (MOS) subject classifications (1970). Primary 57A15, 57A35, 57C55, 57C35; Secondary $57 \mathrm{C30}$.

Key words and phrases. Topological embeddings, codimension 2 cells, 4-space, piecewise linear approximations, locally flat approximations. 
the property that $D \mid U$ can be $\epsilon$-approximated by locally flat PL embeddings for every $\epsilon>0$, then $D$ has the same property.

Corollary. If $D: I^{n-2} \rightarrow M^{n}$ is a PL embedding, then $D$ can be $\epsilon$ approximated by locally flat PL embeddings for every $\epsilon>0$.

3. Sketch of the proof of Theorem 1. Begin by choosing $\epsilon^{\prime}>0$ and a partition $0=a_{k}<a_{k-1}<\cdots<a_{1}=1$ of $I=[0,1]$ such that if $E: I^{2} \rightarrow$ $M^{4}$ is any embedding satisfying (1) $E(x \times I) \subset N_{\epsilon^{\prime}}(D(x \times I))$ for every $x \in I$ and (2) $E\left(I \times\left[a_{i-1}, a_{i}\right]\right) \subset N_{\epsilon^{\prime}}\left(D\left(I \times\left[a_{i-1}, a_{i}\right]\right)\right)$ for each $i$, then $d(D, E)<\epsilon$. One of the sets $D(x \times I)$ or $E(x \times I)$ will be called a fiber. The idea of the proof is to find a sequence of locally flat PL embeddings $E_{0}, \ldots, E_{k}$ so that each fiber of $E_{j}$ is near the corresponding fiber of $D$ for every $j$ and so that $E_{j+1}$ approximates $D$ on one more of the strips $I \times\left[a_{i-1}, a_{i}\right]$ than $E_{j}$ does.

Let $E: I \times 0 \rightarrow M$ be a PL approximation of $D \mid I \times 0$ given by general position. Extend $E$ to $E: I \times I \rightarrow M$ so that each fiber of $E$ is very short. Consider the homotopy of $E(I \times 1)$ to $D(I \times 1)$ obtained by deforming $E(I \times 1)$ to $D(I \times 0)$ and then moving along fibers of $D$. Using general position we can make the track of this homotopy miss $E(I \times 0)$. By [1] there is an ambient isotopy which pushes $E(I \times 1)$ near to $D(I \times 1)$, keeps $E(I \times 0)$ fixed, and moves parallel to fibers of $D$. Let $E_{0}$ be the embedding obtained by composing $E$ and this isotopy.

Let $U$ be a neighborhood of $D\left(I \times\left[0, a_{2}\right]\right)$ and $V$ be a neighborhood of $D\left(I \times\left[a_{2}, a_{1}\right]\right)$. To get $E_{1}$, we want to pull $E_{0}\left(I \times\left[a_{2}, a_{1}\right]\right)$ into $V$ with an isotopy which moves parallel to fibers of $D$ and keeps $E\left(I \times\left[0, a_{2}\right]\right)$ near $U$. Consider the 2-skeleton $U^{2}$ of $U$. By general position, $U^{2} \cap E_{0}\left(I \times\left[a_{2}, a_{1}\right]\right)$ is a finite number of points. Associated with each of the points there is a shadow down to $E\left(I \times a_{2}\right)$. We use the techniques of [2] to push these shadows out of $V$. After this has been done, we can make $E_{0}\left(I \times\left[a_{2}, a_{1}\right]\right) \cap U^{2}=\varnothing$ by simply pushing the $a_{2}$ level out over these points staying right on the disk. Now use [1] again to engulf the dual 1-skeleton of $U$ keeping $E_{0}(I \times 0)$ fixed. The inverse of the engulfing isotopy pulls the image of $I \times\left[a_{2}, a_{1}\right]$ into $V$ and $E_{1}$ is defined as the composition of the maps described. This process is continued inductively.

\section{REFERENCES}

1. R. H. Bing, Vertical general position, Geometric Topology (Proc. Conf., Utah, 1974), Lecture Notes in Math., vol. 438, Springer-Verlag, Berlin and New York, 1975, pp. 16-41. MR 50 \#14751.

2. R. H. Bing and J. M. Kister, Taming complexes in hyperplanes, Duke Math. J. 31 (1964), 491-511. MR 29 \#1626.

3. J. L. Bryant and C. L. Seebeck III, Locally nice embeddings in codimension three, Quart. J. Math. Oxford Ser. (2) 21 (1970), 265-272. MR 44 \#7560.

4. R. H. Fox and J. W. Milnor, Singularities of 2.-spheres in 4-space and cobordism of knots, Osaka J. Math. 3 (1966), 257-267. MR 35 \#2273.

5. G. A. Venema, Approximating disks in 4-space (to appear). 